\title{
RICERCHE ARCHEOBOTANICHE ANALISI DEI CAMPIONI DI TERRA PROVENIENTI DAGLI SCAVI ESEGUITI A SAN POTITO

\author{
ORSOLYA DÁLNOKI
}

\author{
Università degli Studi Lóránd Eötvös, Istituto di Studi Archeologici \\ Múzeum krt. N 4/B, H-1088 Budapest, Ungheria \\ E-mail:
}

\begin{abstract}
Archaaeobotanical research in the Roman villa at San Potito. Partly carbonated seeds testify that barley, millet, lentil, legumes, walnut and strawberry were planted in the territory that belonged to the villa. Grape and cereals, the remains of which were found in a small amount, were probably transported from elsewhere to the inhabitants of the villa located $1000 \mathrm{~m}$ high. This is suggested by the presence of the amphorae as well. Remains of wetland plants and fish scales were recovered by flotation; their presence was due to the vicinity of the Fucine Lake.
\end{abstract}

Keywords: carbonated seeds, barley, millet, lentil, legumes, walnut and strawberry

\section{INTRODUZIONE}

\section{Metodologia}

A San Potito, la prima campionatura archeobotanica è stata effettuata nel settembre del 2001, prelevando da quattro unità stratigrafiche separate da $1 \mathrm{a} 3$ campioni di terreno della quantità da $5 \mathrm{a} 10$ litri. Successivamente fu eseguita sul luogo anche la decantazione. Tale operazione è stata realizzata sulla piccola "piazza" situata sotto la chiesa, utilizzando dopo l'operazione tradizionale di sedimentazione, un secchio, acqua corrente e tre setacci (mm 4-1-0,5). Il materiale rimanente (una miscela di materie organiche ed inorganiche) è stato sottoposto ad essicazione per ottenere un materiale assolutamente asciutto e al fine di prevenire la putrefazione i campioni furono messi in sacchetti di carta. Il selezionamento e l'elaborazione dei campioni furono eseguiti dopo essere tornati in patria.

I campioni di terra uno ad uno sono:

1) 1;-2 sepoltura(?) situata vicino al muro d'epoca romana, forse mescolata a materiale medievale, totale: 10 litri

2)a $m ;-2 /-3$ strato puramente d'epoca romana, piuttosto rosso, bruciato (strato d'incendio), con macchie nere, sparse - della parte superiore provengono $3 \times 10$ litri

2)b $m ;-2 /-3$ continuazione del medesimo strato 10-11 litri

2)c $m ;-2 /-3$ continuazione del medesimo strato

3) riempimento della latrina, terra umida, grigiastra con pezzettini di carbonella bruciata e nera, 5 litri

4) cortile $D / 2 / 3$ - buca 8 litri 


\section{Elaborazione}

Dopo aver pesato i campioni disseccati ha avuto inizio un primo selezionamento costituito dalla divisione dei materiali organici ed inorganici. Le diverse categorie del materiale poi, furono incartate separatamente. In seguito si eseguì il selezionamento fine con la definizione dei detriti macro inclusi. Al fine venne la documentazione.

\section{RISULTATI}

Nel materiale inorganico figuravano tasselli di mosaico e frammenti tessili (?). Tra gli ossi minori abbiamo trovato una quantità sorprendente di squame di pesce. Ogni campione di terra conteneva anche resti organici di piante che costituirono il materiale vero e proprio a partire dai pezzettini di carbonella in stato mal conservato fino ai semi il cui stato di conservazione era, invece, sorprendentemente buono. Meno sorprendente è invece il fatto che, oltre ai detriti carbonificati, nel materiale figuravano anche reperti archeobotanici non carbonificati. Questi semi recenti non possono essere qualificati altrimenti che come frutti della nostra epoca, provenienti dall'ambiente circostante. Nella tavola sono stati indicati con caratteri italici. Un gran numero di semi di fragola conservati in stato umido e rinvenuti nella latrina costituiscono un fenomeno interessante. Si ipotizza che questi reperti, come anche i frammenti di semi di uva, rinvenuti nello stesso luogo sono d'epoca romana.

Materiale vegetale e valutazione

I resti carbonificati ritrovati a San Potito possono essere individuati come rifiuti di cucina fra i quali figuravano semi di cereali, come frumento da seme, avena e orzo, miglio, piante leguminose: lenticchie, vicia ervilia e le diverse specie di veccia (vicia), e anche uva vinicola e noci. La quantità delle piante selvatiche (erbacce) capitate per caso tra il grano era minore, come: il farinello, atripex (una specie appartenente alla famiglia delle Amaranthaceae), festuca, bromus (appartiene alla famiglia delle graminacee), caglio zolfino, poligono concolvio (fallopia

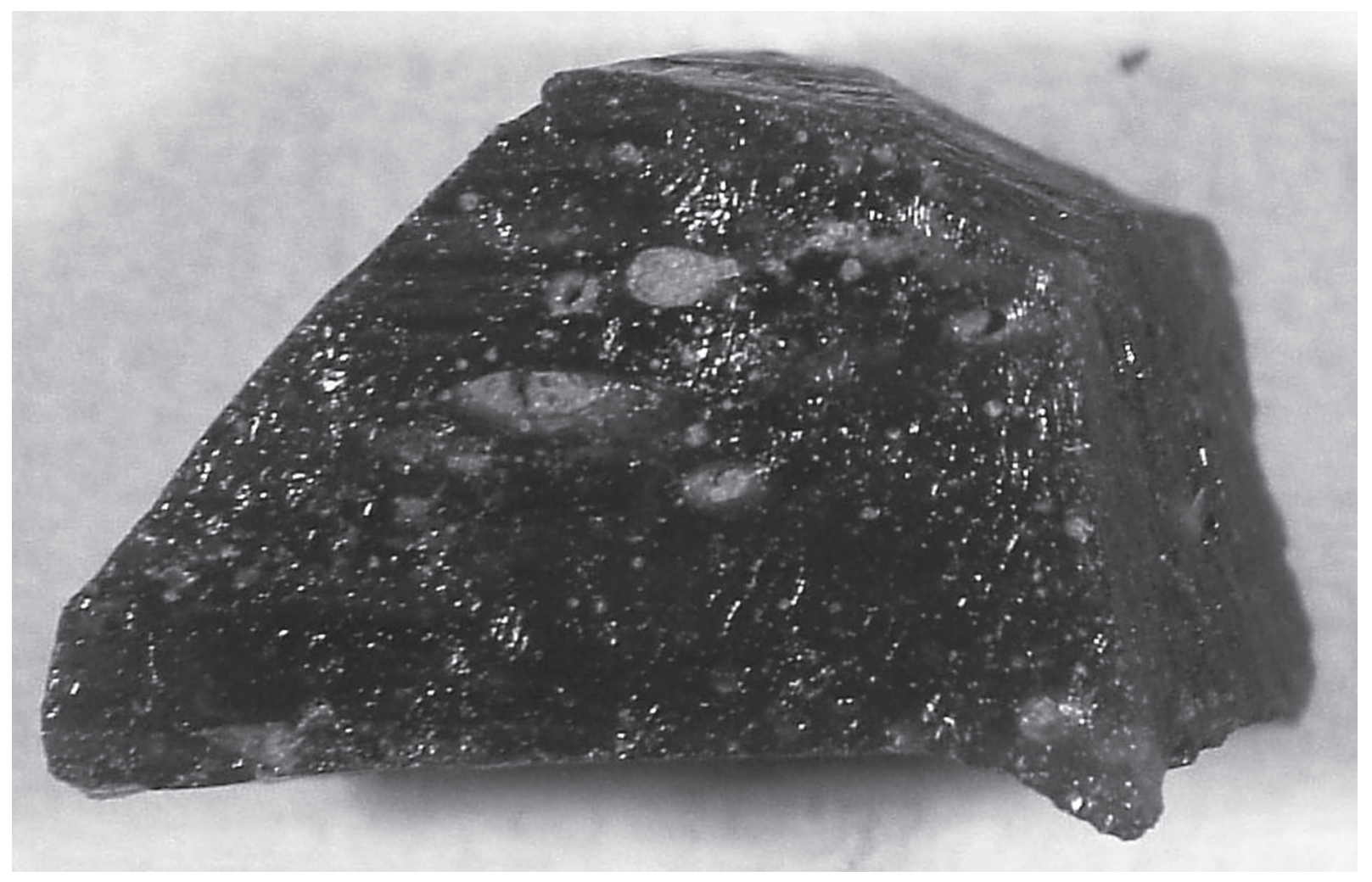

Fig. 1. Frammenti mosaico 
conv.). Probabilmente a seguito dell'impaludamento di alcune zone vennero alla luce anche "piante acquatiche": potamogetonaceae, eleocharis (palustris?). Inoltre la verbena officinalis è un antichissimo rimedio popolare la cui presenza può essere pure attribuita all'epoca romana. Il reperto di maggior interesse e nello stesso tempo il più misterioso potrebbe essere un pinolo carbonificato, ma non si può escludere nemmeno che si tratti del frammento di un seme di ciliegio selvatico.

I campioni uno ad uno:

1) Pezzettini di carbonella, frammenti di malta, lumache, semi recenti, ossi.

2)a Frammenti di carbonella, mosaico, tessuti/pelle (?), reperti vegetali carbonificati: noci, orzo, miglio, sambuca, lenticchie, graminacee, farinello e molti semi recenti non carbonificati.

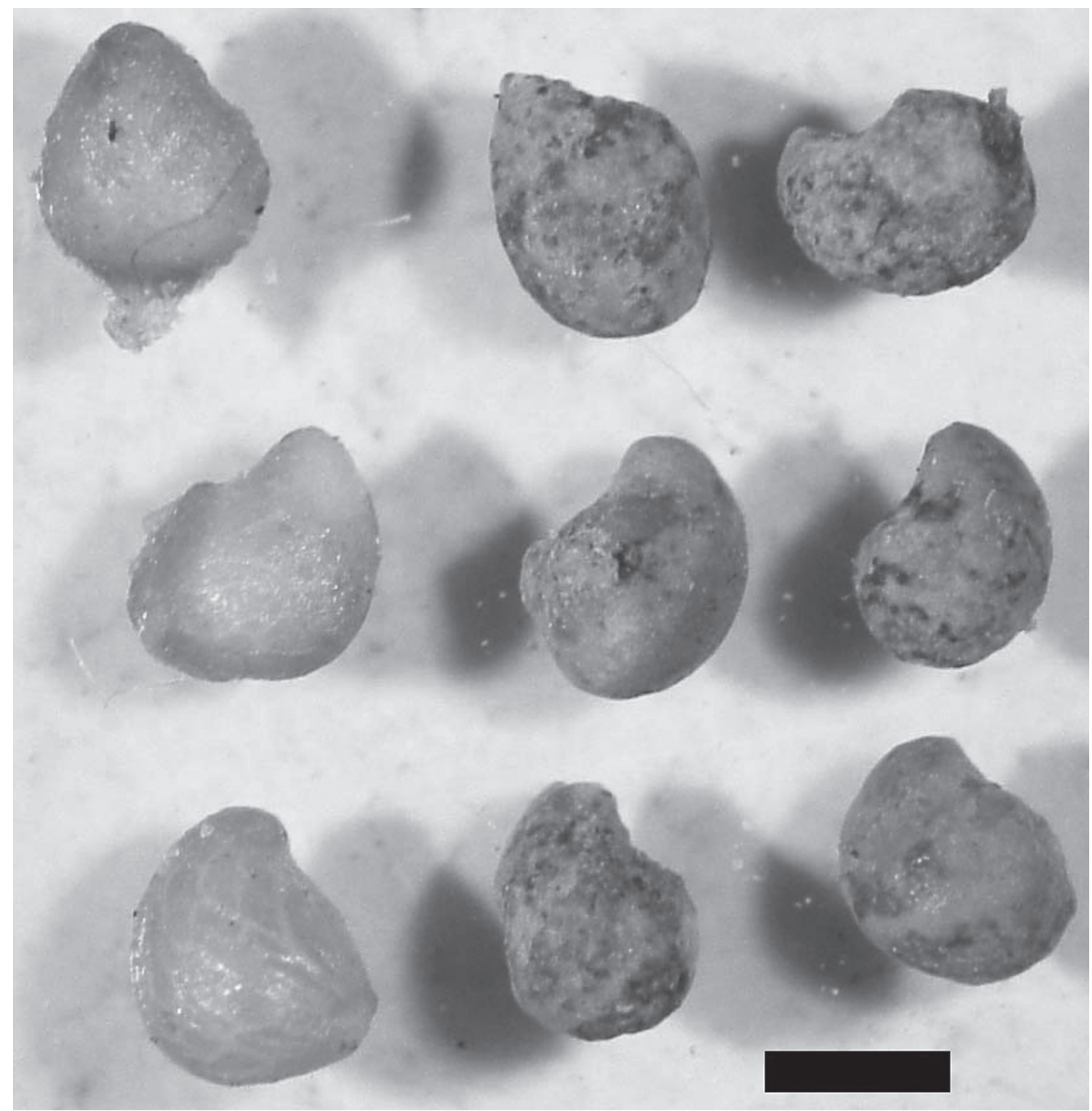

Fig. 2. Semi di fragola 


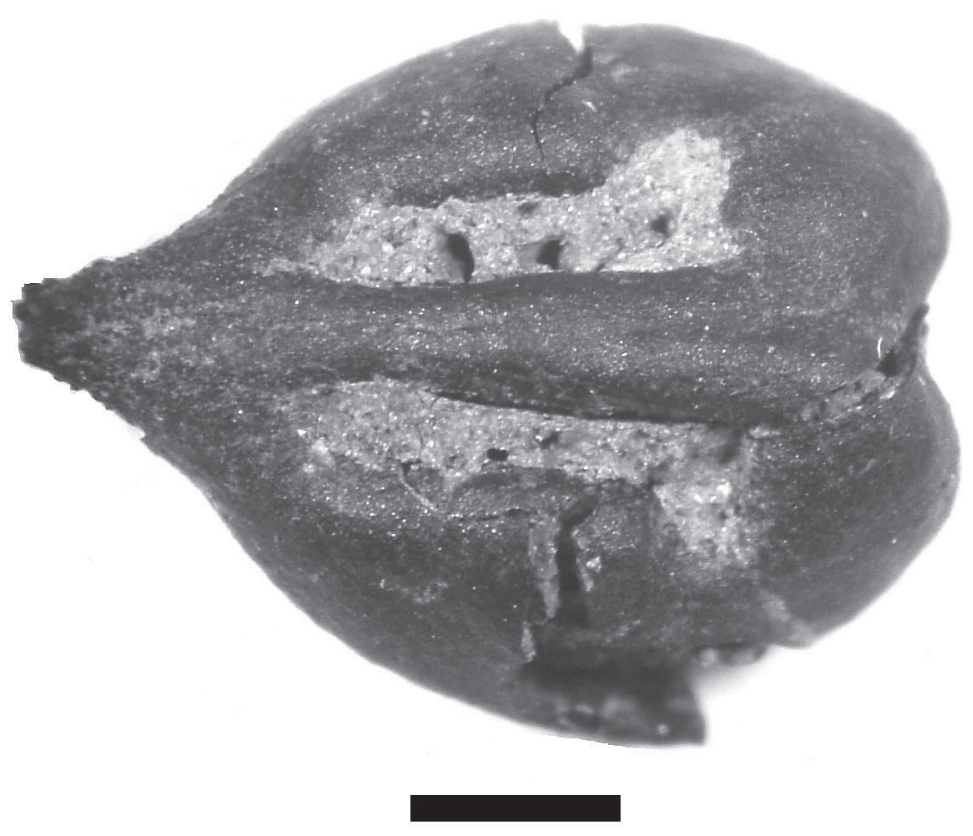

Fig. 3. Seme di uva

2)b Frammenti di carbonella, scaglie di pesce. Resti vegetali carbonificati: noci, uva, lenticchie, avena (frammenti), veccia, vicia sepium, eleocharis (palustris?), verbena, farinello, polygonacee, la cuscuta (un parassite) appartiene alla famiglia delle ranunculacae.

2)c Carbonella, lumache, coprolite, spine. Questo campione contiene il maggior numero di specie vegetali. Orzo, miglio, setaria, frumento, noci, lenticchie, bromus (graminacee), caglio, veccia (vicia), vicia ervilia, potamogetonaceae, pinolo (?), poligono concolvio (fallopia conv.), romice, verbena, caryophylliddae, graminacee.

3) Moltissime scaglie di pesce. Frammenti di carbonella ed un unico frammento di lenticchia (?) carbonificata. L'unico seme di uva non carbonificato e conservatosi in ambiente umido, nonché le varie centinaia di semi di fragola possono essere ritenuti rifiuti umani dell'epoca romana (?).

4) Moltissimi semi recenti e formiche. Pochi reperti carbonificati: noci, atripex, festuca, frammenti di seme di uva, loglio.

\section{RIASSUNTO}

Riassumendo possiamo stabilire che pur avendo trovato entro la villa pochi indizi sospetti dal punto di vista archeobotanico, tuttavia siamo riusciti a eseguire la campionatura prelevando campioni di terra che contenevano piante conservatesi in stato carbonificato o non carbonificato e così, questa fu la prima volta che abbiamo potuto conoscere almeno un piccolo segmento del "regime e delle usanze alimentari" della villa di San Potito. 

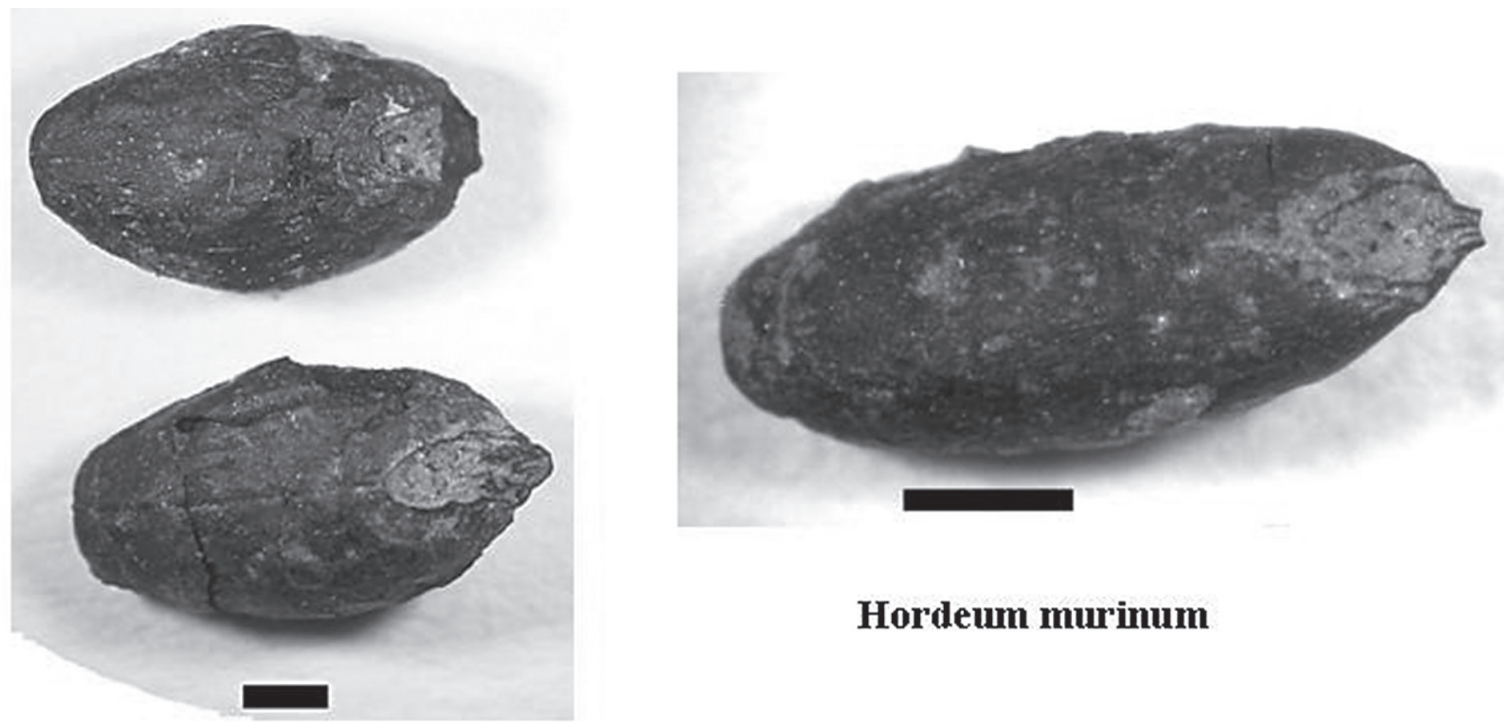

Hordeum murinum

\section{Hordeum vulgare}

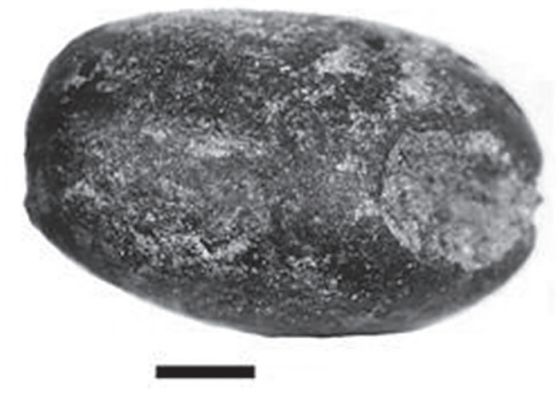

Triticum aestivum

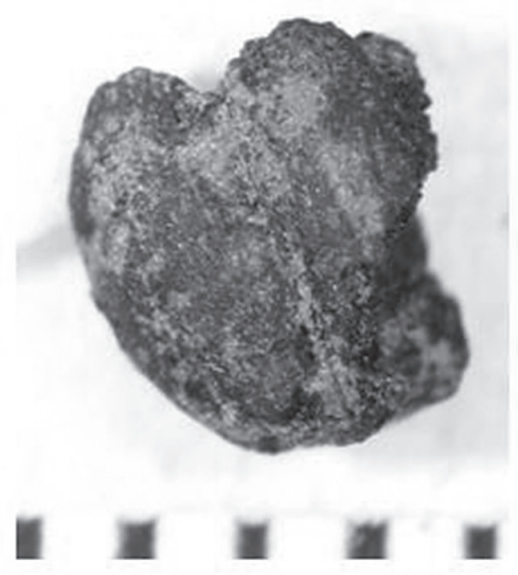

Triticum spec.

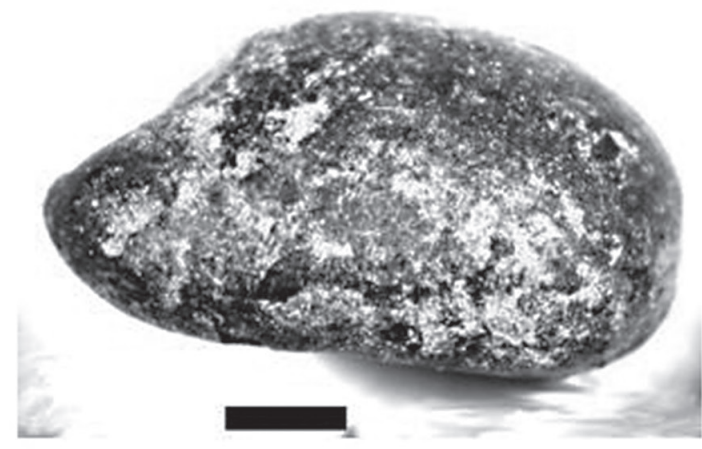

Triticum aestivum

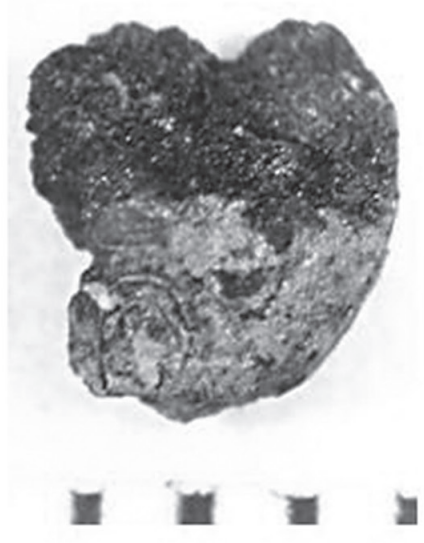

Triticum spec.

Fig. 4. Hordea e diverse specie di triticum 

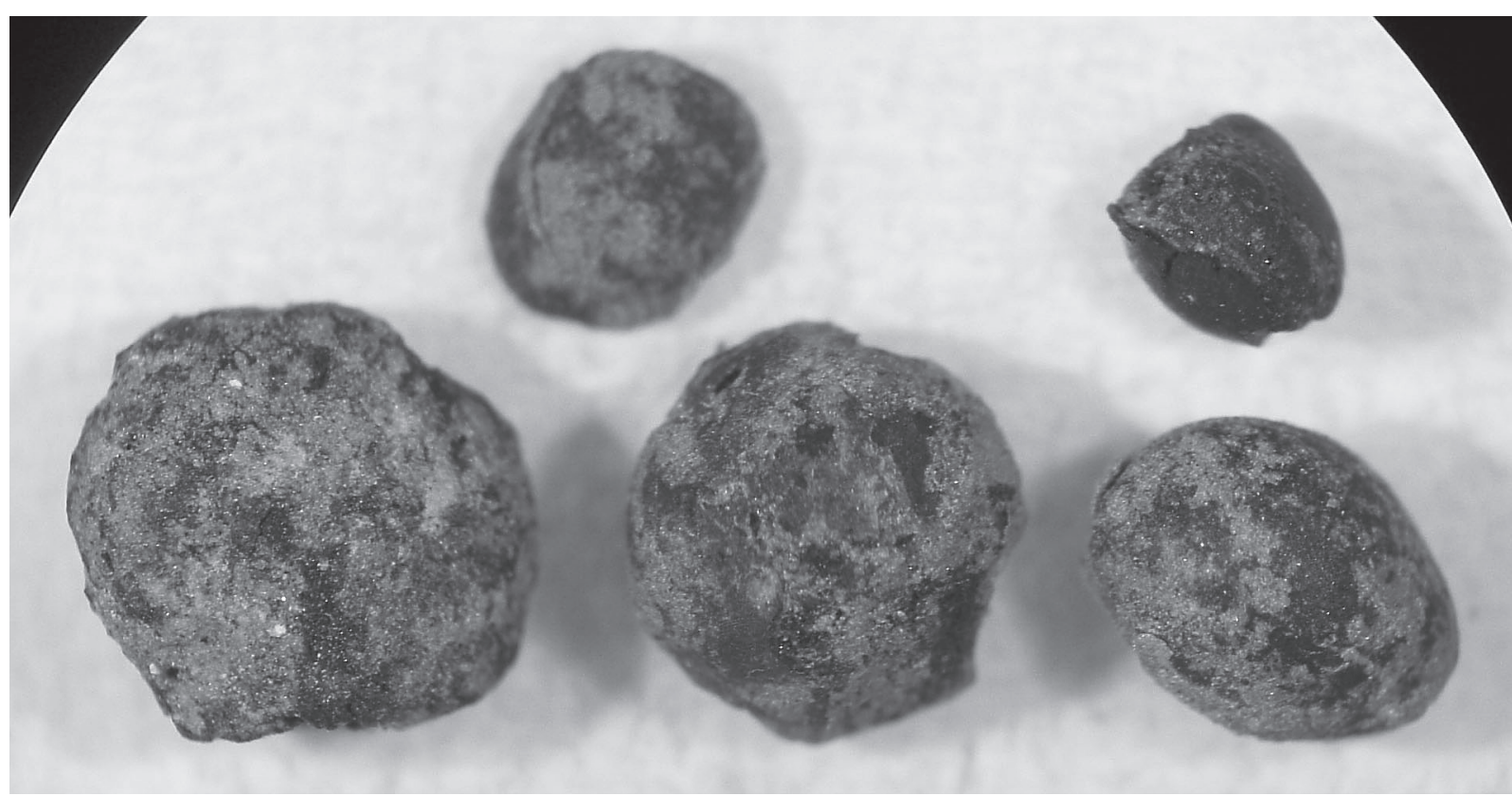

Fig. 5. Pianre leguminose

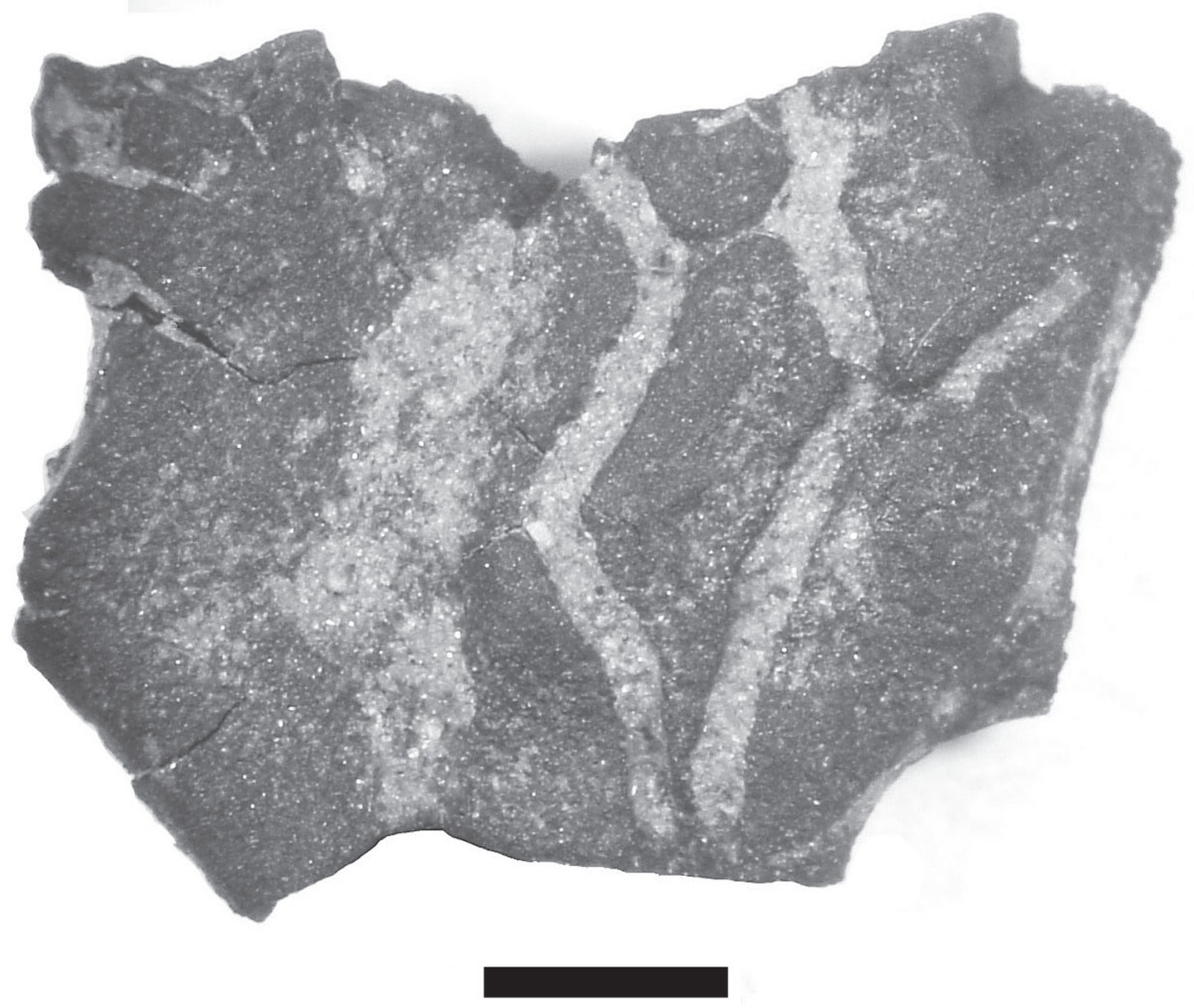

Fig. 6. Conteccino di noce 
San Potito settembre 2001

\begin{tabular}{|c|c|c|c|c|c|c|c|c|c|c|c|c|c|c|c|c|c|c|c|}
\hline & & 1 & & & $2 / \mathbf{a}$ & & & $2 / \mathrm{b}$ & & & $2 / \mathrm{c}$ & & & 3 & & & 4 & & \\
\hline $\begin{array}{l}\text { Quantitŕ prima della } \\
\text { decantazione (1) }\end{array}$ & & 10 & & & 30 & & & 10 & & & 16 & & & 5 & & & 8 & & \\
\hline $\begin{array}{l}\text { Quantitŕ dopo la } \\
\text { decantazione (1) }\end{array}$ & 28,6 & 24,9 & 19,4 & 22 & 52 & 31 & 33 & 60 & 37 & 35 & 43 & 29 & 27 & 22 & 23 & & 18 & 19 & \\
\hline $\begin{array}{l}\text { Diametro del filtro } \\
(\mathrm{mm})\end{array}$ & 4 & 1 & 0,5 & 4 & 1 & 0,5 & 4 & 1 & 0,5 & 4 & 1 & 0,5 & 4 & 1 & 0,5 & 4 & 1 & 0,5 & \\
\hline Mintagyüjtés helye & & \multicolumn{2}{|l|}{ 1: -2} & & \multicolumn{2}{|c|}{$m:-2 /-3$} & & \multicolumn{2}{|c|}{$m:-2 /-3$} & & \multicolumn{2}{|c|}{$m:-2 /-3$} & & \multicolumn{2}{|c|}{ latrina } & & \multicolumn{2}{|l|}{$\mathrm{D} 2 / 3$} & Total \\
\hline Carbonella & $\mathrm{x}$ & $\mathrm{x}$ & $\mathrm{x}$ & $\mathrm{X}$ & $\mathrm{x}$ & $\mathrm{x}$ & $\mathrm{x}$ & & $\mathrm{X}$ & & $\mathrm{x}$ & $\mathrm{x}$ & $\mathrm{x}$ & $\mathrm{X}$ & & $\mathrm{x}$ & $\mathrm{x}$ & & \\
\hline Ossa animali & $\mathrm{x}$ & $\mathrm{x}$ & & & $\mathrm{x}$ & & & & & & $\mathrm{x}$ & & & & & & & & \\
\hline Scagli di pesce & & & & & & & & $\mathrm{x}$ & $\mathrm{x}$ & $\mathrm{x}$ & $\mathrm{x}$ & $\mathrm{x}$ & $\mathrm{X}$ & $\mathrm{x}$ & $\mathrm{x}$ & & & & \\
\hline Lumache & $\mathrm{x}$ & $\mathrm{x}$ & $\mathrm{x}$ & & $\mathrm{x}$ & $\mathrm{x}$ & & & & & $\mathrm{x}$ & & & $\mathrm{x}$ & & & & & \\
\hline indet (non definito) & & $\mathrm{x}$ & & $\mathrm{x}$ & & $\mathrm{X}$ & & $\mathrm{x}$ & $\mathrm{x}$ & & $\mathrm{x}$ & $\mathrm{x}$ & & & & & & $\mathrm{X}$ & \\
\hline Coprolite & & & & & & & & & & & & $\mathrm{x}$ & & & & & & & \\
\hline Rami & & & & & $\mathrm{x}$ & & & & & & (x) & & & & & & & & \\
\hline Avena spec. frgm & & & & & & & & 1 & & & & & & & & & & & 1 \\
\hline Hordeum vulgare & & & & & 5 & & & & & & & & & & & & & & 5 \\
\hline $\begin{array}{l}\text { Hordeum vulgare } \\
\text { frgm }\end{array}$ & & & & & & & & & & & 1 & & & & & & & & 1 \\
\hline Triticum aestivum & & & & & & & & & & & & & & & & & & & 0 \\
\hline Panicum miliaceum & & & & & 3 & & & & & & 1 & & & & & & & & 4 \\
\hline Cerealia frgm & & & & & & & & & & & 3 & & & & & & & & 3 \\
\hline cf Lens culinaris & & & & & 4 & & & 1 & & & 2 & & & & & & & & 7 \\
\hline cf Lens culinaris frgm & & & & & & & & & & & & & & 4 & & & & & 4 \\
\hline Vicia spec. & & & & & & & & & & & 10 & & & & & & & & 10 \\
\hline Vicia spec. frgm & & & & & & & & 7 & & & 2 & & & & & & & & 9 \\
\hline Vicia cf ervilia & & & & & & & & 1 & & & & & & & & & & & 1 \\
\hline Fabaceae frgm & & & & & 4 & & & & & & & & & & & & & & 4 \\
\hline Fabaceae pici & & & & & 2 & & & & & & & 1 & & & & & & & 3 \\
\hline Atriplex spec. & & & & & & & & & & & & & & & & & 1 & & 1 \\
\hline Atriplex spec. frgm & & & & & & & & & & & & & & & & & & 4 & 4 \\
\hline Bromus spec. frgm & & & & & & & & & & & 1 & & & & & & & & 1 \\
\hline Caryophyllaceae & & & & & & & & & & & 1 & & & & & & & & 1 \\
\hline Chenopodiaceae frgm & & & & & & 1 & & & & & & & & & & & & & 1 \\
\hline Chenopodium spec. & & & & & & & & & 1 & & 1 & & & & & & & & 2 \\
\hline Cuscuta spec. & & & & & & & & & 2 & & & & & & & & & & 2 \\
\hline Eleocharis spec. frgm & & & & & & & & 1 & & & & & & & & & & & 1 \\
\hline Fallopia convolvulus & & & & & & & & & & & 1 & & & & & & & & 1 \\
\hline Festuca pratensis & & & & & & 1 & & & & & & & & & & & & 1 & 2 \\
\hline Galium aparine & & & & & & & & & & & 2 & & & & & & & & 2 \\
\hline Galium spec. & & & & & & & & & & & 2 & & & & & & & & 2 \\
\hline Galium spurium & & & & & & & & & & & 3 & & & & & & & & 3 \\
\hline $\begin{array}{l}\text { Pineaceae/Prunus } \\
\text { avium frgm }\end{array}$ & & & & & & & & & & & 1 & & & & & & & & 1 \\
\hline
\end{tabular}




\begin{tabular}{|c|c|c|c|c|c|c|c|c|c|c|c|c|c|c|c|c|c|c|c|}
\hline & & 1 & & & $2 / a$ & & & $2 / b$ & & & $2 / c$ & & & 3 & & & 4 & & \\
\hline Poa spec. & & & & & 1 & & & & & & & & & & & & & 1 & 2 \\
\hline Poaceae & & & & & & & & & 10 & & & 2 & & & & & & & 12 \\
\hline Poaceae frgm & & & & & & 2 & & 1 & & & & & & & & & & & 3 \\
\hline $\begin{array}{l}\text { Polygonum spec. } \\
\text { frgm }\end{array}$ & & & & & & & & 1 & & & & & & & & & & & 1 \\
\hline $\begin{array}{l}\text { Potamogeton cf na- } \\
\text { tans }\end{array}$ & & & & & & & & & & & 1 & & & & & & & & 1 \\
\hline cf Ranunculaceae & & & & & & & & 1 & & & & & & & & & & & 1 \\
\hline Rumex spec. & & & & & & & & & & & 1 & & & & & & & & 1 \\
\hline Setaria/Panicum & & & & & & & & & & & 1 & & & & & & & & 1 \\
\hline cf Verbena officinalis & & & & & & & & & 1 & & & 1 & & & & & & & 2 \\
\hline Juglans frgm & & & & 9 & 3 & & 11 & 34 & & 6 & 14 & 2 & & & & & 6 & & 85 \\
\hline Fragaria & & & & & & & & & & & & & & 500 & 500 & & & & 1000 \\
\hline $\begin{array}{l}\text { Vitis vinifera } \\
\text { ssp. vinifera }\end{array}$ & & & & & & & & 1 & & & & & & & & & & & 1 \\
\hline $\begin{array}{l}\text { Vitis vinifera ssp. } \\
\text { vinifera frgm }\end{array}$ & & & & & & & & & & & & & 1 & & & & & 1 & 2 \\
\hline Euphorbia spec. & & 3 & & & & & & & & & & & & & & & & & 3 \\
\hline Polygonum aviculare & & & & 1 & & & & & & & & & & & & & & & 1 \\
\hline Sambucus spec. & & & & & & 1 & & & & 1 & & & & & & & & & 2 \\
\hline Saponaria & & & & & & & & & & & & & & & & & 1 & & 1 \\
\hline Veronica hederifolia & & & & & & & & & & & & & & & & & 2 & & 2 \\
\hline Papaver cf dubium & & & & & & & & & & & & 1 & & & & & & 2 & 3 \\
\hline Picris hieracioides & & & & & & & & & & & & & & & & & & 2 & 2 \\
\hline Total & 0 & 3 & 0 & 10 & 22 & 5 & 11 & 49 & 14 & 7 & 48 & 7 & 1 & 504 & 500 & 0 & 10 & 11 & 1202 \\
\hline
\end{tabular}

\section{BIBLIOGRAFIA}

AMOURETTI 1992

AmouretTi 1988

BAAS 1974

BAAS 1982

BEIJERINCK 1947

BERTSCH 1949

ERMÉNYI 1977

FLACH 1990

FURGER 1985

GyUlai 1988
= M. C. Amouretti: Agriculture in Ancient Greece. In: B. Wells (ed.): Agriculture in Ancient Greece. Proceedings of the Seventh International Symposium at the Swedish Institute at Athens, 16-17 May, 1990. Acta Instituti Atheniensis Regni Sueciae. Series in 4o, 42. Stockholm 1992, 77-86.

= M. C. AmouretTi: La viticulture antique: Contraintes et choix téchniques. REA 90/1-2 (1988) 5-17.

= J. BAAS: Kultur- und Wildpflanzenreste aus einem römischen Brunnen von Rottweil-Altstadt. FBBW 1 (1974) 373-416.

= J. BAAS: Kultur- und Nutzpflanzen aus römischen Siedlungsgruben in Nidderau-Heldenbergen. Ein Beitrag zur Geschichte unserer Kulturpflanzen. SaalburgJb 38 (1982) 110-119.

= W. BEIJERINCK: Zadenatlas, der Nederlandische Flora. Wageningen 1947.

= K. F. BERTSCH: Geschichte unserer Kulturpflanzen. Stuttgart 1949.

= M. P. ERMÉNYI: Forrástanulmány a régészeti korokból származó csonthéjas gyümölcs leletekről Közép-Európában (Quellenstudie über den Steinfruchtfunden in Mitteleuropa aus Archäologischen Zeiten). MMMK 1975/1977 (1977) 135-165.

= D. FlaCH: RömischeAgrargeschichte. München 1990.

= A. R. Furger: Vom Essen und Trinken im römischen Augst: Kochen, Essen und Trinken im Spiegel einiger Funde. ASchw 8 (1985) 168-187.

= F. GyUlai: Obst undWeinfundeaus der Arpadenzeit (10. Jahrhundert) in Fonyód-Bélatelep am Balaton. In: H. Küster (Hrsg.): Der prähistorische Mensch und seine Umwelt. Festschrift Udelgard Körber-Grohne zum 65. Geburtstag. Forschungen und Berichte zur Vor- und Frühgeschichte in Baden-Württemberg 31 Stuttgart 1988, 395-402. 
GYULAI 1995

HARTYÁNYI-NOVÁKI 1975

JACOMET 1986

JASHEMSKI 1979

KNÖRZER 1966

KNÖRZER 1970

Körber-GROHNE 1979

KROLL 1994

KÜSTER H. 1994

LENZ 1966

NETOLICZKY 1942

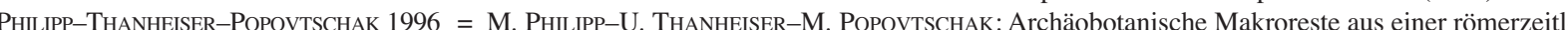

SCHERMANN 1966

SCHNEIDER 1988

= F. GYULAI: The analytical study of carbonized grain remains from the Lake Balaton Region (Hungary). Annali di Botanica (Roma) 53 (1995) 251-260.

= B. P. HARTYÁNYI-Gy. NovÁKI: Samen- und Fruchtfunde in Ungarn von der Neusteinzeit bis zum 18. Jahrhundert. AgrSz [Historia Rerum Rusticarum] 17. Suppl. Budapest 1975, 1-22.

= S. JACOMET: Verkohlte Pflanzenreste aus einem römischen Grabmonument beim Augster Osttor. Jahresberichte aus Augst und Kaiseraugst 6 (1986) 7-53.

= W. F. JASHEMSKI: The gardens of Pompeii: Herculaneum and the villas destroyed by Vesuvius. New York 1979 .

= K-H. KNÖRZER: Über Funde römischer Importfrüchte in Novaesium (Neuß/Rh.). BonnerJb 166 (1966) 433-443.

= K-H. KNÖRZER: Römerzeitliche Pflanzenfunde aus Neuss. Novaesium 4. Limes Forschungen 10. Berlin 1970 .

= U. KÖRBER-GroHNE: Nutzpflanzen und Umwelt im römischen Germanien. Schriften Limesmus. Aalen 21. Stuttgart 1979.

= H. KROLL: Pflanzliche Großreste aus einer Latrine vom Auerberg. In: G. Ulbert: Der Auerberg. I. Topographie, Forschungsgeschichte und Wallgrabungen. München 1994, 199-211.

$=$ H. KÜSTLER: Botanische Untersuchungen zur Landwirtschaft in den Rhein-Donau-Provinzen vom 1. bis zum 5. Jahrhundert nach Chr. In: H. Bender-H. Wolff (Hrsg.): Rural Settlement and Farming in the Rhine-Danube Provinces of the Roman Empire. Proceedings of an International Colloquy at Passau, April 16-21, 1991. 2.: Text. Espelkamp 1994, 21-36.

= H. O. LENZ: Botanik der alten Griechen und Römer. Wiesbaden 1966.

= F. NetoliczKy: Unser Wissen von den alten Kulturpflanzen Mitteleuropas. BRGK 20 (1942) 14-76. ichen Latrine in Carnuntum (Ende 2./Anfang 3. Jh.n. Chr.). Bericht. Miben? Megjel. hely, oldalszám $=$ Sz. Schermann: Magismeret [olaszul, v. idegen nyelvü kivonat címe] I-II. Budapest 1996.

$=$ H. SCHNEIDER: Verkohlte pflanzliche Makroreste aus Grabungen in Augst und Kaiseraugst. Kulturund Wildpflanzenfunde als Informationsquelle über Römerzeit. Jahresberichte aus Augst und Kaiseraugst 9 (1988) 287 